\title{
Higgs Boson Gluon-Fusion Production in QCD at Three Loops
}

\author{
Charalampos Anastasiou, ${ }^{1}$ Claude Duhr, ${ }^{2,3,{ }^{*}}$ Falko Dulat, ${ }^{1}$ Franz Herzog, ${ }^{4}$ and Bernhard Mistlberger ${ }^{1}$ \\ ${ }^{1}$ Institute for Theoretical Physics, ETH Zürich, 8093 Zürich, Switzerland \\ ${ }^{2}$ CERN Theory Division, 1211 Geneva 23, Switzerland \\ ${ }^{3}$ Center for Cosmology, Particle Physics and Phenomenology (CP3), Université Catholique de Louvain, \\ 1348 Louvain-La-Neuve, Belgium \\ ${ }^{4}$ Nikhef, Science Park 105, 1098 XG Amsterdam, The Netherlands
}

(Received 20 March 2015; published 27 May 2015)

\begin{abstract}
We present the cross section for the production of a Higgs boson at hadron colliders at next-to-next-tonext-to-leading order $\left(\mathrm{N}^{3} \mathrm{LO}\right)$ in perturbative QCD. The calculation is based on a method to perform a series expansion of the partonic cross section around the threshold limit to an arbitrary order. We perform this expansion to sufficiently high order to obtain the value of the hadronic cross at $\mathrm{N}^{3} \mathrm{LO}$ in the large topmass limit. For renormalization and factorization scales equal to half the Higgs boson mass, the $\mathrm{N}^{3} \mathrm{LO}$ corrections are of the order of $+2.2 \%$. The total scale variation at $\mathrm{N}^{3} \mathrm{LO}$ is $3 \%$, reducing the uncertainty due to missing higher order QCD corrections by a factor of 3 .
\end{abstract}

DOI: 10.1103/PhysRevLett.114.212001

PACS numbers: $12.38 . \mathrm{Bx}$

The success of the Large Hadron Collider (LHC) experiments in the exploration and interpretation of phenomena at the $\mathrm{TeV}$ scale is due, on the one hand, to amazing experimental and technological advances and, on the other hand, to extraordinary progress in perturbative QCD. In particular, the discovery of the Higgs boson [1] by the LHC experiments has initiated an era of precision studies of the properties of the Higgs boson, where precise theory predictions for Higgs observables play an indispensable role.

The inclusive gluon-fusion cross section is a prototypical example of a theoretical input for the interpretation of the experimental observations. It enters not only into the extraction of the Higgs-boson couplings from the measurements, but it could also play an important role in identifying deviations from the standard model predictions in Higgs physics. Unfortunately, the theory predictions for the inclusive cross section are plagued by significant theoretical uncertainties. Scale variations at next-to-next-to-leading order (NNLO) indicate that missing higher order effects are of the order of $\pm 9 \%$ at LHC energies [2,3], and the size of this uncertainty is comparable to the experimental uncertainty from LHC run $1[4,5]$. Hence, with a few more years of data taking the theoretical uncertainty will be dominant, demanding an update of the current theoretical predictions.

In this context, a vigorous effort has recently been made to compute the inclusive gluon-fusion cross section at nextto-next-to-next-to-leading order $\left(\mathrm{N}^{3} \mathrm{LO}\right)$ in perturbative QCD. The cross section at $\mathrm{N}^{3} \mathrm{LO}$ receives contributions from many different building blocks, all of which have

Published by the American Physical Society under the terms of the Creative Commons Attribution 3.0 License. Further distribution of this work must maintain attribution to the author(s) and the published article's title, journal citation, and DOI. been computed over the last years, at least partially. The three-loop corrections to Higgs production in gluon fusion have been obtained in Ref. [6], and the corrections from the emission of an additional parton at one or two loops were computed in Refs. [7-11]. In order to obtain a finite result, appropriate ultraviolet and infrared counterterms need to be included [12-14]. While all of these contributions had been computed in full generality, contributions from the emission of two partons at one loop and three partons at tree level had only been computed in an approximate manner. In particular, for these contributions the first two terms in the expansion around threshold could be obtained [15-18], confirming previous results for logarithmically enhanced terms in the cross section [19] and resulting in the complete computation of the inclusive gluon-fusion cross section at $\mathrm{N}^{3} \mathrm{LO}$ in the soft-virtual $[17,18,20]$ and next-to-soft approximations [21]. Owing to the universality of soft emissions, the previous results have sparked various new results for QCD processes at $\mathrm{N}^{3} \mathrm{LO}$ in the soft-virtual approximation [22].

Despite this progress, the soft-virtual and next-to-soft approximations are insufficient to make reliable predictions for the cross section, owing to a slow convergence of the threshold expansion [21]. In this Letter we close this gap, and we present the gluon-fusion Higgs production cross section at $\mathrm{N}^{3} \mathrm{LO}$ in perturbative QCD. We emphasize that this is the first ever complete computation of a cross section at $\mathrm{N}^{3} \mathrm{LO}$ at a hadron collider.

We will describe the main result of our computation in this Letter, while a detailed account of the mathematical and computational methods will be presented elsewhere. Here, it suffices to say that we work in the framework of reverse unitarity [23,24], and we perform a complete reduction of the cross section to master integrals, without any approximations. For the double- and triple-emission 
contributions at $\mathrm{N}^{3} \mathrm{LO}$, we can derive differential equations satisfied by the master integrals [23-25], which we solve as generalized power series around the threshold limit. In this way, we obtain at least 37 terms in the threshold expansion of each master integral. Additional terms can be obtained at the expense of computing time. An important part of our computation has been the evaluation of the boundary conditions that are needed for solving the differential equations for the master integrals. Many of the boundary conditions required in this project had already been derived in the context of the soft-virtual and next-to-soft results [15-18,21]. Using similar techniques, we have computed the remaining few unknown boundary conditions for master integrals that start to be relevant only at a high order in the threshold expansion.

Having at our disposal the complete set of master integrals as expansions around the threshold limit, we can easily obtain the cross sections at $\mathrm{N}^{3} \mathrm{LO}$ for all partonic channels contributing to Higgs production via gluon fusion. The partonic cross sections are related to the hadronic cross section at the LHC through the integral

$$
\sigma=\sum_{i, j} \int d x_{1} d x_{2} f_{i}\left(x_{1}, \mu_{f}\right) f_{j}\left(x_{2}, \mu_{f}\right) \hat{\sigma}_{i j}\left(z, \mu_{r}, \mu_{f}\right),
$$

where the summation indices $i, j$ run over the parton flavors in the proton, $f_{i}$ are parton densities, and $\hat{\sigma}_{i j}$ are partonic cross sections. Furthermore, we define $z=m_{H}^{2} / s$, where $m_{H}$ is the mass of the Higgs boson and $\sqrt{s}$ is the partonic center-of-mass energy, related to the hadronic center-ofmass energy $\sqrt{S}$ through $s=x_{1} x_{2} S$. The renormalization and factorization scales are denoted by $\mu_{r}$ and $\mu_{f}$. We work in an effective theory approach where the top quark is integrated out. The effective Lagrangian describing the interaction of the Higgs boson and the gluons is

$$
\mathcal{L}_{\text {eff }}=-\frac{C}{4} H G_{\mu \nu}^{a} G^{a \mu \nu},
$$

where $H$ is the Higgs field, $G_{\mu \nu}^{a}$ is the gluon field strength tensor, and $C$ is the Wilson coefficient, known up to nextto-next-to-next-to-next-to leading order $\left(\mathrm{N}^{4} \mathrm{LO}\right)$ [26]. We expand the partonic cross sections into a perturbative series in the strong coupling constant evaluated at the scale $\mu_{r}$

$$
\hat{\sigma}_{i j}=\hat{\sigma}_{0}\left[\delta_{i g} \delta_{j g} \delta(1-z)+\sum_{\ell=1}^{\infty}\left(\frac{\alpha_{s}\left(\mu_{r}\right)}{\pi}\right)^{\ell} \hat{\sigma}_{i j}^{(\ell)}\right] .
$$

In this expression $\hat{\sigma}_{0}$ denotes the leading order (LO) cross section, and the terms through NNLO in the above expansion have been computed in Refs. [23,27,28]. The main result of this Letter is the result for the $\mathrm{N}^{3} \mathrm{LO}$ coefficient, corresponding to $\ell=3$ in Eq. (3), for all possible parton flavors in the initial state. We cast the $\mathrm{N}^{3} \mathrm{LO}$ coefficients in the form

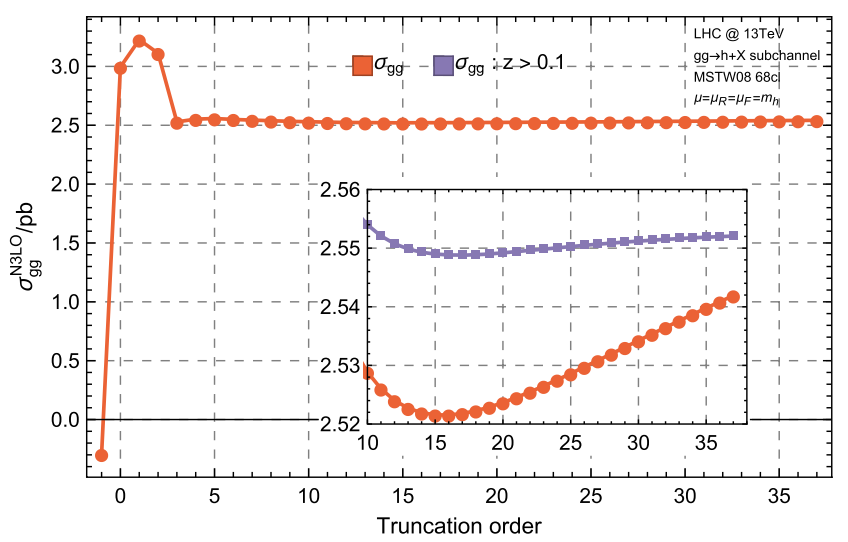

FIG. 1 (color online). The $\mathrm{N}^{3} \mathrm{LO}$ correction from the $g g$ channel to the hadronic cross section as a function of the truncation order $N$ in the threshold expansion for the scale choice $\mu=m_{H}$. The inlay shows that the convergence improves when the high-energy tail $(z<0.1)$ of the hadronic integral in Eq. (1) is removed.

$$
\hat{\sigma}_{i j}^{(3)}=\lim _{N \rightarrow \infty} \hat{\sigma}_{i j}^{(3, N)}
$$

where we introduce the truncated threshold expansions defined by

$$
\hat{\sigma}_{i j}^{(3, N)}=\delta_{i g} \delta_{j g} \hat{\sigma}_{\mathrm{SV}}^{(3)}+\sum_{n=0}^{N} c_{i j}^{(n)}(1-z)^{n} .
$$

Here, $\hat{\sigma}_{\mathrm{SV}}^{(3)}$ denotes the soft-virtual cross section at $\mathrm{N}^{3} \mathrm{LO}$ of Refs. $[17,18,20]$ and $N=0$ is the next-to-soft approximation of Ref. [21]. Using our method for the threshold expansion of the master integrals, we were able to determine the $c_{i j}^{(n)}$ analytically up to at least $n=30$. Note that at any given order in the expansion these coefficients are polynomials in $\log (1-z)$. While this approach does not cast the partonic cross sections in a closed analytic form, we argue that it yields the complete result for the value of the hadronic cross section. In Fig. 1 we show the contribution of the partonic cross section coefficients $\mathrm{N}^{3} \mathrm{LO}$ to the hadronic cross section for a proton-proton collider with a $13 \mathrm{TeV}$ center-of-mass energy as a function of the truncation order $N$. We use NNLO MSTW2008 [29] parton densities and a value for the strong coupling at the mass of the $Z$ boson of $\alpha_{s}\left(m_{Z}\right)=0.117$ as the initial value for the evolution, and we set the factorization scale to $\mu_{f}=m_{H}=125 \mathrm{GeV}$. We observe that the threshold expansion stabilizes starting from $N=4$, leaving a negligible truncation uncertainty for the hadronic cross section thereafter. We note, though, that we observe a very small, but systematic, increase of the expansion in the range $N \in[15,37]$, as illustrated in Fig. 1. We have observed that a similar behavior is present in the threshold expansion at NNLO. The systematic increase originates from values of the partonic cross section at very small $z$. Indeed, this increase appears only in the contributions to the hadronic 


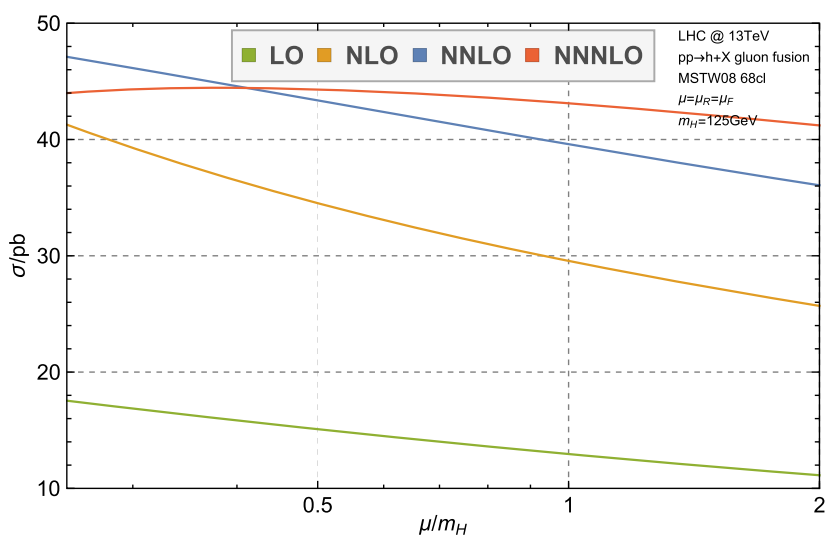

FIG. 2 (color online). Scale $\mu=\mu_{r}=\mu_{f}$ variation of the gluonfusion cross section at all perturbative orders through $\mathrm{N}^{3} \mathrm{LO}$ for $m_{H}=125 \mathrm{GeV}$. We use NNLO MSTW2008 [29] parton densities and $\alpha_{s}\left(m_{Z}\right)=0.117$ with $\mathrm{N}^{3} \mathrm{LO}$ evolution at all perturbative orders.

cross section integral for values of $z<0.1$. It is natural that the terms of the threshold expansion computed here do not furnish a good approximation of the hadronic integral in the small $z$ region due to the divergent high energy behavior of the partonic cross sections [30]. However, it is observed that this region is suppressed in the total hadronic integral and for $z<0.1$ contributes less than $0.4 \%$ of the total $\mathrm{N}^{3} \mathrm{LO}$ correction. The same region at next-to-leading order (NLO) and NNLO, where analytic expressions valid for all regions are known, is similarly suppressed. We therefore believe that the uncertainty of our computation for the hadronic cross section due to the truncation of the threshold expansion is negligible (less than $0.2 \%$ ).

In Fig. 2 we present the hadronic gluon-fusion Higgs production cross section at $\mathrm{N}^{3} \mathrm{LO}$ as a function of a common renormalization and factorization scale $\mu=$ $\mu_{r}=\mu_{f}$. We use NNLO parton densities and N3LO evolution of the strong coupling not only in N3LO, but also in LO, NLO, and NNLO. We observe a significant reduction of the sensitivity of the cross section to the scale $\mu$. Inside a range $\mu \in\left[m_{H} / 4, m_{H}\right]$ the cross section at $\mathrm{N}^{3} \mathrm{LO}$ varies in the interval $[-2.7 \%,+0.3 \%]$ with respect to the cross section value at the central scale $\mu=m_{H} / 2$. For comparison, we note that the corresponding scale variation at NNLO is about $\pm 9 \%[2,3]$. This improvement in the precision of the Higgs cross section is a major accomplishment due to our calculation and will have a strong impact

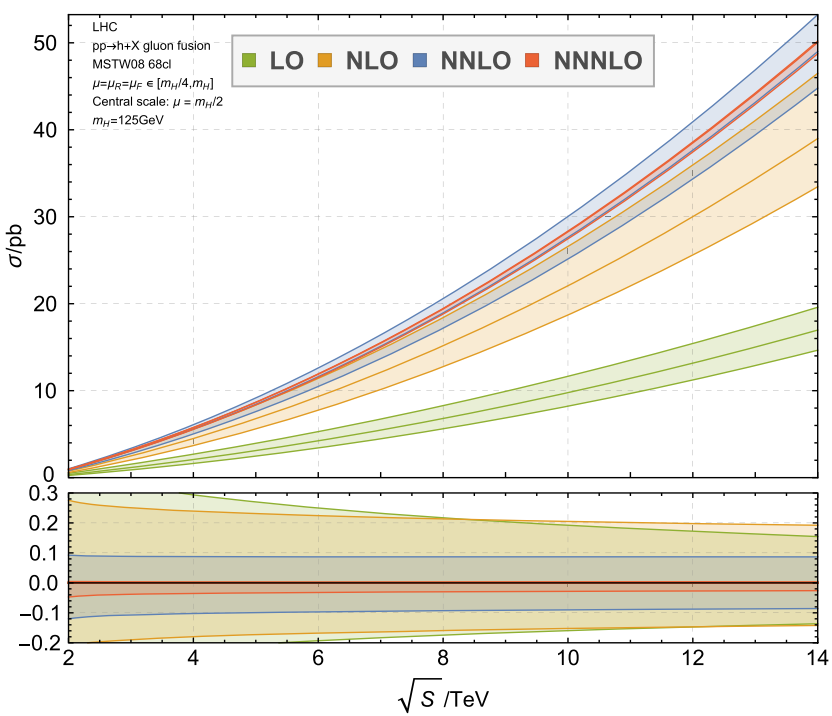

FIG. 3 (color online). The LHC gluon-fusion cross section through $\mathrm{N}^{3} \mathrm{LO}$ for a common scale $\mu \in\left[m_{H} / 4, m_{H}\right]$ as a function of the center-of-mass energy $\sqrt{S}$. In the lower panel the cross section is normalized to its value at $\mu=m_{H} / 2$.

on future measurements of Higgs-boson properties. Furthermore, even though for the scale choice $\mu=$ $m_{H} / 2$ the $\mathrm{N}^{3} \mathrm{LO}$ corrections change the cross section by about $+2.2 \%$, this correction is captured by the scale variation estimate for the missing higher order effects of the NNLO result at that scale. We illustrate this point in Fig. 3, where we present the hadronic cross section as a function of the hadronic center-of-mass energy $\sqrt{S}$ at the scale $\mu=m_{H} / 2$. We observe that the $\mathrm{N}^{3} \mathrm{LO}$ scale uncertainty band is included within the NNLO band, indicating that the perturbative expansion of the hadronic cross section is convergent. However, we note that for a larger scale choice, e.g., $\mu=m_{H}$, the convergence of the perturbative series is slower than for $\mu=m_{H} / 2$.

In Table I we quote the gluon-fusion cross section in the effective theory at $\mathrm{N}^{3} \mathrm{LO}$ for different LHC energies. The perturbative uncertainty is determined by varying the common renormalization and factorization scale in the interval $\left[m_{H} / 4, m_{H}\right]$ around $m_{H} / 2$ and in the interval $\left[m_{H} / 2,2 m_{H}\right]$ around $m_{H}$.

Given the substantial reduction of the scale uncertainty at $\mathrm{N}^{3} \mathrm{LO}$, the question naturally arises whether other sources of theoretical uncertainty may contribute at a similar level. In the remainder of this Letter we briefly comment on this

TABLE I. The gluon-fusion cross section in the effective theory as a function of the (proton-proton) collider energy. in the interval $\left[m_{H} / 4, m_{H}\right]$ around $\mu=m_{H} / 2$ and in the interval $\left[m_{H} / 2,2 m_{H}\right]$ around $\mu=m_{H}$.

\begin{tabular}{lccccc}
\hline \hline$\sigma / \mathrm{pb}$ & $2 \mathrm{TeV}$ & $7 \mathrm{TeV}$ & $8 \mathrm{TeV}$ & $13 \mathrm{TeV}$ & $14 \mathrm{TeV}$ \\
\hline$\mu=m_{H} / 2$ & $0.99_{-4.65 \%}^{+0.43 \%}$ & $15.31_{-3.08 \%}^{+0.31 \%}$ & $19.47_{-2.99 \%}^{+0.32 \%}$ & $44.31_{-2.64 \%}^{+0.31 \%}$ & $49.87_{-2.61 \%}^{+0.32 \%}$ \\
$\mu=m_{H}$ & $0.94_{-7.35 \%}^{+4.87 \%}$ & $14.84_{-5.27 \%}^{+3.18 \%}$ & $18.90_{-5.02 \%}^{+3.08 \%}$ & $43.14_{-4.45 \%}^{+2.71 \%}$ & $48.57_{-4.24 \%}^{+2.68 \%}$ \\
\hline \hline
\end{tabular}


issue, leaving a more detailed quantitative study for future work.

First, we note that given the small size of the $\mathrm{N}^{3} \mathrm{LO}$ corrections compared to NNLO, we expect that an estimate for the higher-order corrections at $\mathrm{N}^{4} \mathrm{LO}$ and beyond can be obtained from the scale variation uncertainty. Alternatively, partial $\mathrm{N}^{4} \mathrm{LO}$ results can be obtained by means of factorization theorems for threshold resummation. However, we expect that the insight from resummation on the $\mathrm{N}^{4} \mathrm{LO}$ soft contributions is only qualitative given the importance of next-to-soft, next-to-next-to-soft, and purely virtual contributions observed at $\mathrm{N}^{3} \mathrm{LO}$, as seen in Fig. 1.

Electroweak corrections to Higgs production have been calculated through two loops in Ref. [31], and estimated at three loops in Ref. [32]. They furnish a correction of less than $+5 \%$ to the inclusive cross section. Thus, they are not negligible at the level of accuracy indicated by the scale variation at $\mathrm{N}^{3} \mathrm{LO}$ and need to be combined with our result in the future. Mixed QCD-electroweak or purely electroweak corrections of even higher order are expected to contribute at the subpercent level and should be negligible.

Next, we have to comment on our assumption that the top quark is infinitely heavy and can be integrated out, see Eq. (2). Moreover, we assumed that all other quarks have a zero Yukawa coupling. Finite quark mass effects are important, but it is sufficient that they are included through NLO or NNLO. Indeed, finite quark-mass effects have been computed fully through NLO in QCD [27], while subleading top-quark mass corrections have been computed at NNLO systematically as an expansion in the inverse topquark mass [33]. In these references it was observed that through NLO finite quark mass effects amount to about $8 \%$ of the $K$ factor. At NNLO, the known $1 / m_{\text {top }}$ corrections affect the cross section at the $\sim 1 \%$ level. A potentially significant contribution at NNLO that has not yet been computed in the literature originates from diagrams with both a top and bottom quark Yukawa coupling. Assuming a similar perturbative pattern as for top-quark only diagrams in the effective theory (2), higher-order effects could be of the order of $2 \%$. We thus conclude that the computation of the top-bottom interference through NNLO is highly desired in the near future.

Finally, the computation of the hadronic cross section relies crucially on the knowledge of the strong coupling constant and the parton densities. After our calculation, the uncertainty coming from these quantities has become dominant. Further progress in the determination of parton densities must be anticipated in the next few years due to the inclusion of LHC data in the global fits and the impressive advances in NNLO computations, improving the theoretical accuracy of many standard candle processes.

To conclude, we have presented in this Letter the computation of the gluon-fusion Higgs production cross section through $\mathrm{N}^{3} \mathrm{LO}$ in perturbative QCD. While a thorough study of the impact of electroweak and quark mass effects is left for future work, the remaining theoretical uncertainty on the inclusive Higgs production cross section is expected to be reduced by roughly a factor of two, which will bring important benefits in the study of the properties of the Higgs boson at LHC run 2. Besides its direct phenomenological impact, we believe that our result is also a major advance in our understanding of perturbative $\mathrm{QCD}$, as it opens the door to push the theoretical predictions for large classes of inclusive processes to $\mathrm{N}^{3} \mathrm{LO}$ accuracy, like Drell-Yan production, associated Higgs production, and Higgs production via bottom fusion. Moreover, on the more technical side, our result constitutes the first independent validation of the gluon splitting function at NNLO [14], because the latter is required to cancel all the infrared poles in the inclusive cross section. In addition, we expect that the techniques developed throughout this work are not restricted to inclusive cross sections, and it should be possible to extend them to certain classes of differential distributions, like rapidity distributions for Drell-Yan and Higgs production, thereby paving the way to a new era of precision QCD.

We are grateful to Elisabetta Furlan, Thomas Gehrmann, and A. Lazopoulos for our collaboration on the many aspects of the Higgs cross-section $\mathrm{N}^{3} \mathrm{LO}$ project that are not covered in this Letter. We thank A. Lazopoulos in particular for an independent implementation of our results in IHIXS and numerical comparisons. The research was supported by the Swiss National Science Foundation (SNF) under Contract No. 200021-143781 and the European Commission through the ERC grants "IterQCD," "HEPGAME," and "MathAm".

*On leave from the "Fonds National de la Recherche Scientifique" (FNRS), Belgium.

[1] G. Aad et al. (ATLAS Collaboration), Phys. Lett. B 716, 1 (2012); S. Chatrchyan et al. (CMS Collaboration), Phys. Lett. B 716, 30 (2012).

[2] C. Anastasiou, S. Bühler, F. Herzog, and A. Lazopoulos, J. High Energy Phys. 04 (2012) 004.

[3] C. Anastasiou, S. Bühler, F. Herzog, and A. Lazopoulos, J. High Energy Phys. 12 (2011) 058.

[4] V. Khachatryan et al. (CMS Collaboration), arXiv: 1412.8662.

[5] G. Aad et al. (ATLAS Collaboration), Phys. Rev. D 90, 112015 (2014).

[6] P. A. Baikov, K. G. Chetyrkin, A. V. Smirnov, V. A. Smirnov, and M. Steinhauser, Phys. Rev. Lett. 102, 212002 (2009); T. Gehrmann, E. W. N. Glover, T. Huber, N. Ikizlerli, and C. Studerus, J. High Energy Phys. 06 (2010) 094.

[7] C. Anastasiou, C. Duhr, F. Dulat, F. Herzog, and B. Mistlberger, J. High Energy Phys. 12 (2013) 088.

[8] W. B. Kilgore, Phys. Rev. D 89, 073008 (2014).

[9] T. Gehrmann, M. Jaquier, E. W. N. Glover, and A. Koukoutsakis, J. High Energy Phys. 02 (2012) 056.

[10] C. Duhr and T. Gehrmann, Phys. Lett. B 727, 452 (2013); Y. Li and H. X. Zhu, J. High Energy Phys. 11 (2013) 080. 
[11] C. Duhr, T. Gehrmann, and M. Jaquier, J. High Energy Phys. 02 (2015) 077; F. Dulat and B. Mistlberger, arXiv: 1411.3586.

[12] O. V. Tarasov, A. A. Vladimirov, and A. Y. Zharkov, Phys. Lett. 93B, 429 (1980); S. A. Larin and J. A. M. Vermaseren, Phys. Lett. B 303, 334 (1993); T. van Ritbergen, J. A. M. Vermaseren, and S. A. Larin, Phys. Lett. B 400, 379 (1997); M. Czakon, Nucl. Phys. B710, 485 (2005).

[13] C. Anastasiou, S. Bühler, C. Duhr, and F. Herzog, J. High Energy Phys. 11 (2012) 062; M. Höschele, J. Hoff, A. Pak, M. Steinhauser, and T. Ueda, Phys. Lett. B 721, 244 (2013); S. Bühler and A. Lazopoulos, J. High Energy Phys. 10 (2013) 096.

[14] S. Moch, J. A. M. Vermaseren, and A. Vogt, Nucl. Phys. B688, 101 (2004); B691, 129 (2004).

[15] C. Anastasiou, C. Duhr, F. Dulat, and B. Mistlberger, J. High Energy Phys. 07 (2013) 003.

[16] H. X. Zhu, J. High Energy Phys. 02 (2015) 155.

[17] C. Anastasiou, C. Duhr, F. Dulat, E. Furlan, T. Gehrmann, F. Herzog, and B. Mistlberger, Phys. Lett. B 737, 325 (2014).

[18] Y. Li, A. von Manteuffel, R. M. Schabinger, and H. X. Zhu, Phys. Rev. D 90, 053006 (2014).

[19] S. Moch and A. Vogt, Phys. Lett. B 631, 48 (2005); E. Laenen and L. Magnea, Phys. Lett. B 632, 270 (2006); N. A. Lo Presti, A. A. Almasy, and A. Vogt, Phys. Lett. B 737, 120 (2014); D. de Florian, J. Mazzitelli, S. Moch, and A. Vogt, J. High Energy Phys. 10 (2014) 176.

[20] Y. Li, A. von Manteuffel, R. M. Schabinger, and H. X. Zhu, Phys. Rev. D 91, 036008 (2015).

[21] C. Anastasiou, C. Duhr, F. Dulat, E. Furlan, T. Gehrmann, F. Herzog, and B. Mistlberger, J. High Energy Phys. 03 (2015) 091.

[22] T. Ahmed, M. Mahakhud, N. Rana, and V. Ravindran, Phys. Rev. Lett. 113, 112002 (2014); T. Ahmed, M. K. Mandal, N. Rana, and V. Ravindran, Phys. Rev. Lett. 113, 212003 (2014); T. Ahmed, N. Rana, and V. Ravindran, J. High Energy Phys. 10 (2014) 139; T. Ahmed, M. K. Mandal, N. Rana, and V. Ravindran, J. High Energy Phys. 02 (2015) 131; M. C. Kumar, M. K. Mandal, and V. Ravindran, J. High Energy Phys. 03 (2015) 037; S. Catani, L. Cieri, D. de Florian, G. Ferrera, and M. Grazzini, Nucl. Phys. B888, 75 (2014).
[23] C. Anastasiou and K. Melnikov, Nucl. Phys. B646, 220 (2002).

[24] C. Anastasiou and K. Melnikov, Phys. Rev. D 67, 037501 (2003); C. Anastasiou, L. J. Dixon, and K. Melnikov, Nucl. Phys. B, Proc. Suppl. 116, 193 (2003); C. Anastasiou, L. J. Dixon, K. Melnikov, and F. Petriello, Phys. Rev. Lett. 91, 182002 (2003); C. Anastasiou, L. J. Dixon, K. Melnikov, and F. Petriello, Phys. Rev. D 69, 094008 (2004).

[25] A. V. Kotikov, Phys. Lett. B 259, 314 (1991); 267, 123 (1991).

[26] K. G. Chetyrkin, B. A. Kniehl, and M. Steinhauser, Nucl. Phys. B510, 61 (1998); Y. Schroder and M. Steinhauser, J. High Energy Phys. 01 (2006) 051; K. G. Chetyrkin, J. H. Kuhn, and C. Sturm, Nucl. Phys. B744, 121 (2006).

[27] D. Graudenz, M. Spira, and P. M. Zerwas, Phys. Rev. Lett. 70, 1372 (1993); S. Dawson, Nucl. Phys. B359, 283 (1991); A. Djouadi, M. Spira, and P. M. Zerwas, Phys. Lett. B 264, 440 (1991); M. Spira, A. Djouadi, D. Graudenz, and P. M. Zerwas, Nucl. Phys. B453, 17 (1995); R. Harlander and P. Kant, J. High Energy Phys. 12 (2005) 015; C. Anastasiou, S. Beerli, S. Bucherer, A. Daleo, and Z. Kunszt, J. High Energy Phys. 01 (2007) 082; U. Aglietti, R. Bonciani, G. Degrassi, and A. Vicini, J. High Energy Phys. 01 (2007) 021; R. Bonciani, G. Degrassi, and A. Vicini, J. High Energy Phys. 11 (2007) 095; C. Anastasiou, S. Bucherer, and Z. Kunszt, J. High Energy Phys. 10 (2009) 068.

[28] R. V. Harlander and W. B. Kilgore, Phys. Rev. Lett. 88, 201801 (2002); V. Ravindran, J. Smith, and W. L. van Neerven, Nucl. Phys. B665, 325 (2003).

[29] A. D. Martin, W. J. Stirling, R. S. Thorne, and G. Watt, Eur. Phys. J. C 63, 189 (2009).

[30] F. Hautmann, Phys. Lett. B 535, 159 (2002).

[31] S. Actis, G. Passarino, C. Sturm, and S. Uccirati, Nucl. Phys. B811, 182 (2009); S. Actis, G. Passarino, C. Sturm, and S. Uccirati, Phys. Lett. B 670, 12 (2008); U. Aglietti, R. Bonciani, G. Degrassi, and A. Vicini, Phys. Lett. B 595, 432 (2004).

[32] C. Anastasiou, R. Boughezal, and F. Petriello, J. High Energy Phys. 04 (2009) 003.

[33] R. V. Harlander and K. J. Ozeren, J. High Energy Phys. 11 (2009) 088; A. Pak, M. Rogal, and M. Steinhauser, J. High Energy Phys. 02 (2010) 025. 